\title{
Numerical investigation of the Wave Disk Micro-Engine concept
}

\author{
Janusz Piechna ${ }^{1}$ and David Dyntar ${ }^{2}$ \\ ${ }^{1}$ Warsaw University of Technology \\ Institute of Aeronautics and Applied Mechanics \\ Ul. Nowowiejska 24, 00-665 Warsaw, Poland \\ E-mail:jpie@meil.pw.edu.pl \\ ${ }^{2}$ Swiss Federal Institute of Technology Zurich \\ Physikstrasse 3, 8092 Zurich, Switzerland
}

\begin{abstract}
This paper presents results of numerical investigation, based on two-dimensional model, of a wave disk micro-engine concept taking into account full set of unsteady, centrifugal, Corriolis forces existing in such configuration. Even in two-dimensional simplification, the engine model derived guarantees conservation of mass flow through the simulated combustion chamber. A series of different geometrical configurations is analyzed. Investigation concentrates on the pure unsteady wave engine configuration. The waveengine design investigation presented in recent paper focused on two main problems: realization of gas compression and simultaneously a torque generation. Compression performance has been confirmed in almost any considered configuration but the torque generation was much below the expectations. Results of numerical simulations are presented in form of pressure, temperature and velocity distributions as well torque variations.
\end{abstract}

\section{INTRODUCTION}

Many groups of researchers have focused on the design of micro turbine engines in recent years, Akbari (2004), Epstein (2003), Frechette (2000), Iancu (2005), Piechna (2004, 2005, 2006), Ribaud (2005), Schneider (2005). The range of applications of small turbo engine electric generators is very wide.

Reduction in dimensions and reduction of parts number results in sophisticated simplicity of micro turbo engines in comparison with bigger counterparts. The pace of development seems to be accelerating nowadays.

The idea of the wave engine has been known for many years, see Hörler (1969). The first successful realization of wave engine construction is the wave engine developed by Pearson (1985) in mid-1950s. The very short rotor has helical channels, which change the direction of gas flow, generating shaft work. The engine worked successfully for several hundreds hours in a wide range of operating conditions showing, at low rotational speed, higher efficiency than a comparable conventional turbo engine of those times. The engine had a complicated porting system for realization of single compression and twostage decompression. Two versions of engine have been known. The smaller one had a rotor of about $6 \mathrm{~cm}(2.5$ ") while the second had a larger rotor diameter of about 23 $\mathrm{cm}$ (9"). The larger engine operating in the range of 3000 to $18000 \mathrm{rpm}$ showed that the main principle of operation based on unsteady flows cannot be the only way of operation in such a wide range of rotational speeds. The larger engine produced up to $26 \mathrm{~kW}(35 \mathrm{hp})$ at its design point, with a peak temperature of $1070{ }^{\circ} \mathrm{C}$ and thermal efficiency of about $10 \%$. Accidentally destroyed and never rebuilt, it is known as the most successful wave rotor machine built to date.

Another construction, called shock wave engine, was proposed by the Weber (1995). Taking into account of construction details the machine could be rather called turbo-engine topped by the wave rotor.

The idea of the wave disk engine was considered by Piechna (2005 a,b,c, 2006 a,b). Using a one-dimensional code, numerical models of a single-step and two-step compression-decompression wave engine were build, which showed a rather narrow range of stable operation. The preliminary predictions of the engine efficiency seemed to be promising, however.

\section{STEADY AND UNSTEADY COMPRESSION.}

The major benefit of the application of unsteady-flow phenomena in the construction of flow machines is their potential to generate much greater pressure increase than those obtained in steady-state flow devices. Comparison between the pressure increase generated by a shock wave and possible isentropic compression of gas having the same Mach number is shown in Fig. 1. Assuming the flow of gas with known Mach number $M$, gas can be stopped (compressed) in steady way realizing the energy equation, presented in the following form

$\frac{p}{p_{0}}=\left(1+\frac{k-1}{2} M^{2}\right)^{-\frac{k}{k-1}}$

with stagnation pressure $-p_{0}$, static pressure - $p$,

specific heat ratio $-k$.

Air can be also compressed in unsteady way by the moving upstream shock wave leaving behind the stagnant 
air. Pressure jump generated by the shock wave moving upstream can be calculated from relation

$\frac{p_{2}}{p_{1}}=1+\frac{2 k}{k+1}\left(M_{s}^{2}-1\right)$ where $M_{s}$ - is the shock wave

Mach number calculated from formula

$M=\frac{2}{k+1}\left(M_{s}-\frac{1}{M_{s}}\right)$

Clearly, fluids with the same flow velocity can be compressed much more strongly in non-steady processes than in a steady process.

\section{PHYSICAL PRINCIPLES OF PRESSURE WAVE ROTOR OPERATION}

The simplest physical model of the unsteady compression can be explained on the basis of a tube closed on the right-hand side by a valve and equipped a piston on the left-hand side. Initially, the air inside the tube has an

Pressure ratio

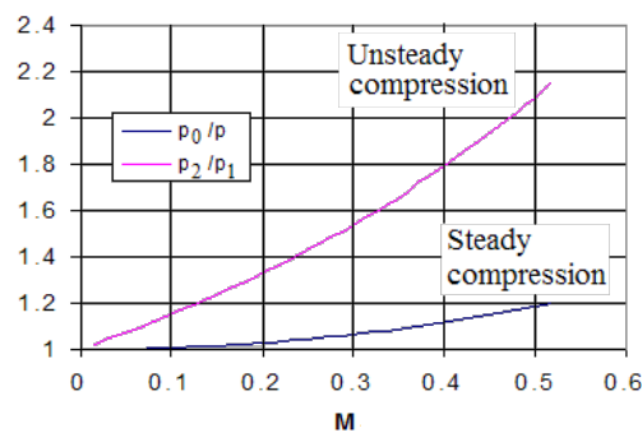

Fig. 1. Comparison of pressure ratios realizable in different compression processes.

ambient pressure and temperature. The fast motion of the piston into the tube starts the unsteady compression. The accelerating piston generates in front of it a set of compression waves coalescing into a shock wave in some distance. Between the piston and the shock wave position there is a dynamically compressed air of a higher temperature, which results in the unsteady compression process. In practical application, a heavy solid piston is exchanged by the light hot exhaust gases.

The principle of the unsteady charger operation is based on the difference in the propagation velocities of the compression (shock) wave and of the contact surface between interacting gases. The contact surface corresponds to the surface of the piston being the prototype of the air to exhaust gas charger. Typically the compression wave is mowing at a speed of $350-400 \mathrm{~m} / \mathrm{s}$ in contrast to the slower $(100 \mathrm{~m} / \mathrm{s})$ motion of the air-togas contact surface. Air can be additionally compressed by the compression wave reflected from the closed righthand side end of the pipe.

The part of the compressed gas located between the actual position of the reflected compression wave and the position of the contact surface can be effectively used after rapid opening the valve on the right-hand side of the pipe. The main disadvantage of this type of compression is its limited, rather short duration of unsteady process. The valve should be closed before it is reached by the exhaust gas contact surface.

The state diagram presented in Fig. 2 corresponding to the steady and unsteady compression-decompression processes shows that in unsteady flows small velocity changes generate great pressure changes, whereas great pressure changes generate only small velocity changes.

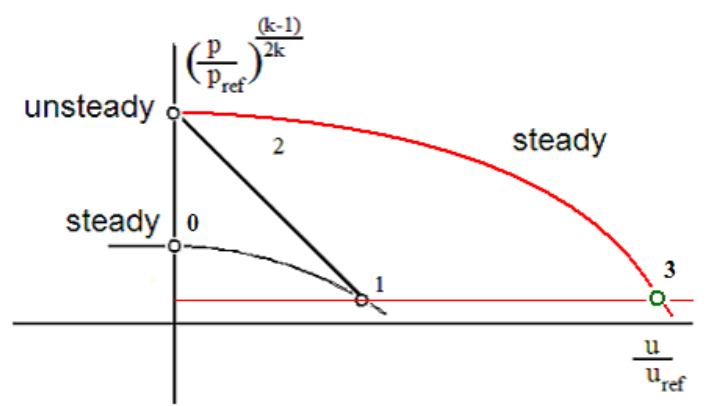

Fig. 2. State diagram presenting comparison of the steady and unsteady compression-decompression processes.

The intention of this figure is to show that while unsteady compression is useful the unsteady decompression is not so effective.

\section{WAVE ENGINE IDEA}

From the thermodynamic point of view, the main advantages of the wave engine are the self-cooling features of the wave process. They allow using higher temperatures in the combustion chamber, thus increasing the engine power and efficiency. In Fig. 3 a temperatureentropy diagram is presented showing thermodynamic advantages of the unsteady process in wave engine in comparison to the steady process in turbine engine.

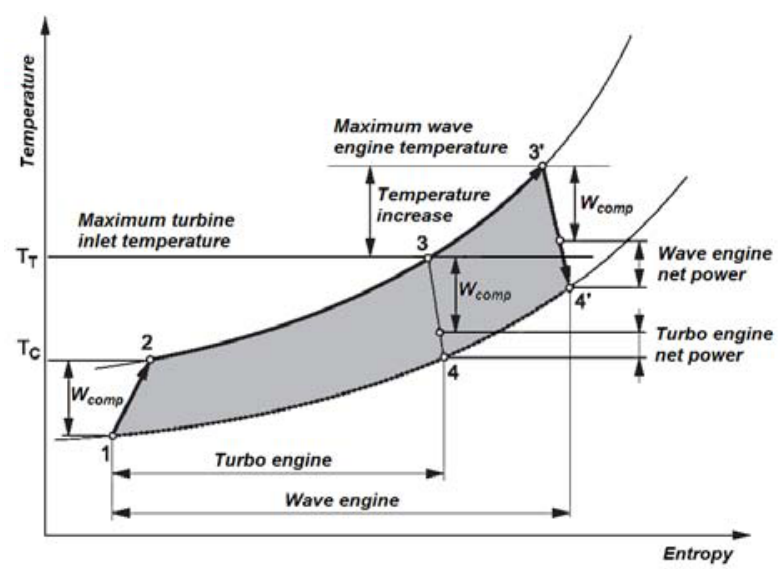

Fig. 3. Temperature-entropy diagram for the wave engine and comparable turbo engine.

While the described thermodynamic idea is clear and simple, its practical realization of such process is not so easy.
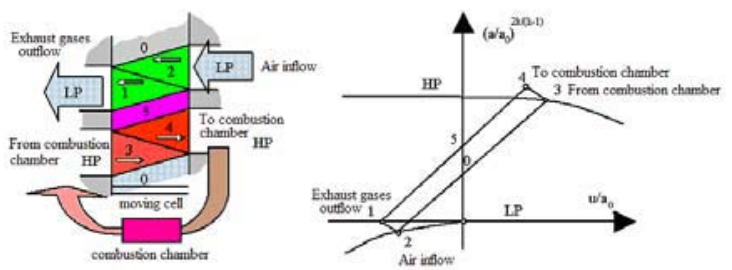

a)

b)

Fig. 4. Wave pattern and state diagram for the basic configuration of the wave engine. 
The main part of the wave engine is a set of compressiondecompression channels called cells. Typically, such cells are distributed axially forming the rotor rotating between two flat cases with sets of ports. During rotation, ends of the cells periodically enter into contact with different ports.

Wave engine design and operation can be explained by the scheme shown in Fig. 4, which presents the wave pattern on the physical plane and in the corresponding state diagram. Figure 4 a) shows a developed rotor with single cell and its pertaining set of ports. Legends in the figure explain the role of each port. Figure $4 \mathrm{~b}$ ) shows the state diagram, with the numbered points describing pressure and velocity corresponding to zones in physical plane. An operation cycle starts when hot gases at high pressure leaving the combustion chamber compress the air in shock wave separating the areas 0 and 3 . The shock wave reflected from the right, closed end of the cell than moves to the left, leaving behind the air compressed enough to be delivered to the combustion chamber (area 4 ). The reflected shock wave between the areas 3 and 4 reaches the left cell end at time when the hot gas port is closed, generating the expansion wave between areas 4 and 5. Clearly, the pressure in area 5 is lower than in combustion chamber. Opening the low-pressure outflow port generates the expansion wave starting the scavenging process (removal of the hot gases from the cell). In areal relatively high velocity can be observed. After reflection from the right hand side cell end the expansion wave between areas 1 and 2 opens the inflow of the fresh air ventilating the cell. The reflected expansion wave is reaching the left end of the cell in phase then the port is closed generating compression wave between areas 2 and 0 . The cycle of operation is closed and can be repeated. Considered scheme shows only the paths of the main waves (compression and expansion) giving the overall information about pressures and velocities existing temporarily inside the cells. One can notice the inflow and outflow velocities, which can be used for torque generation. Using the inclined ducts delivering air or gas a tangential component of reaction of the flow can be expected.

One of expected and recognized problems is scavenging process. The most difficult physical process is the removal of exhaust gases from the compression and decompression chambers and their refilling by the fresh air. Typically, unsteady expansion process increases the exhaust gases outflow velocity and induces inflow of the fresh gas.

Two main processes taking place in the wave engine can be considered. The first is unsteady compression and decompression. The second is torque generation due to the momentum change. The idea of micro turbo-engine made in silicon wafer has opened many new possibilities. Existing prototypes of gas turbine engines, made of stacked silicon wafers, have low efficiency but a potentially very high power-to-weight ratio. Unfortunately the commonly used axial geometry of wave machines cannot be directly applied to MEMS technology.

In 2004 was proposed by Piechna et al. (2004) a new geometry of the wave machine based on the radial cell configuration. In contrast to the axial position of the cells in conventional wave rotor, the additional action of centrifugal and Corriolis forces can be realized in radial geometry. The basic scheme of the wave engine has not been change but some new features of the wave disk can be considered. The main problem with the axial wave rotor was related to bad properties of the scavenging process. It was expected that use of the radial configuration could improve scavenging due to centrifugal forces.

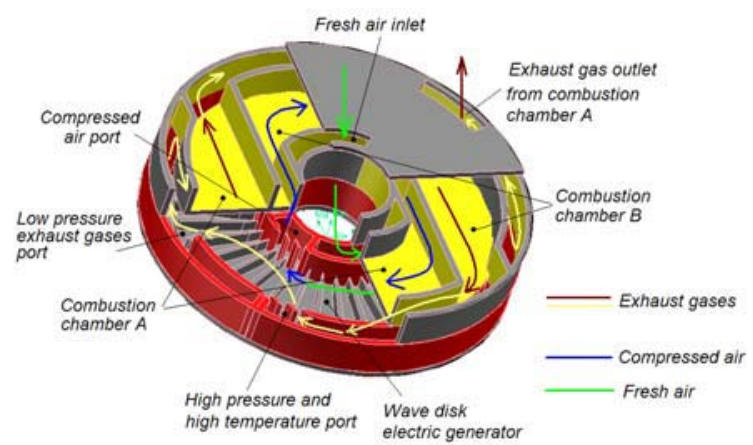

Fig. 5. Possible technical realization of the wave-disk engine idea

The possible construction of wave engine is presented in Fig. 5. Fig. 6 shows the pressure distribution for the single stage wave engine. For clarity, on the left side of figure typical wave diagram is presented, with real pressure distribution in radial configuration shown on the right side. A similar type of visualization of the temperature distribution is depicted in Fig. 7. Data presented in Figs. 6 and 7 are simulation results of unsteady processes based on a one-dimensional model by Piechna (2006).

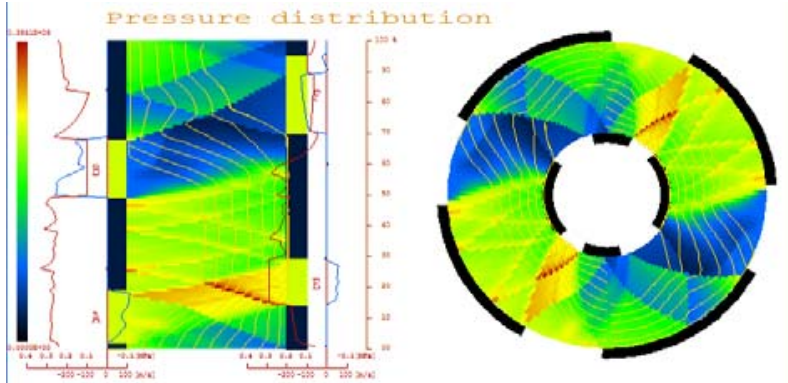

Fig. 6. Pressure distribution for the wave-disk engine (results of 1-D calculation)

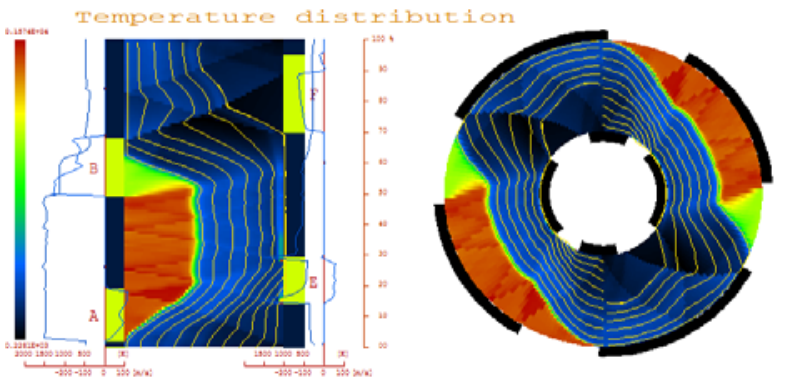

Fig. 7. Temperature distribution for the wave-disk engine (results of 1-D calculation)

\section{DESCRIPTION OF RECENT NUMERICAL MODEL}

In many numerical models considered by Piechna (2005a) the flow parameters at the device ports are assumed or taken from experiments. The main problem with such kinds of boundary conditions is possible loss of 
mass flow conservation between the compressed air port and high-pressure hot gases port. In the case of the wave engine model, this problem is critical.

In the paper of Piechna (2005c, 2006b) based on the onedimensional code model; in order to close the flow loop the compressed air port was connected with the model of combustion chamber. The model of constant heat flux was applied. Compressed air heated inside the combustion chamber was then redirected to the hot gas port. In that way the same mass of gas was made to pass through the compressed air window and hot gas window. Under such assumptions, temperature and pressure inside the combustion chamber were a function of flow rate. Through this the process was stabilized during simulation. Compressible fluid model with Spalart-Allmaras turbulence model were used and solved with the densitybased solver. Pressure inlet and pressure outlet type of boundary conditions have been applied on external boundaries. Interface type of boundary condition has been used between the stator and rotor parts of device. Time step and number of internal iterations have been chosen to fulfill the accuracy requirements (residuals less than 0.001).

In recent simulations based on the two-dimensional model available in FLUENT, it was necessary to solve the problem of the port connections outside the rotor. The main difficulty was caused by the position of some ports inside the rotor and some outside of the rotor. Initially the model base on the recirculation boundary conditions were used, but eventually it was exchanged for a more direct and exact model of geometrically overlapping but logically not interfering zones. Although the code was two-dimensional it was possible, based only on topological relations, to prepare the geometrical connection between proper ports located on both sides of the disk, regardless of geometrical restrictions as is shown in Fig. 8.

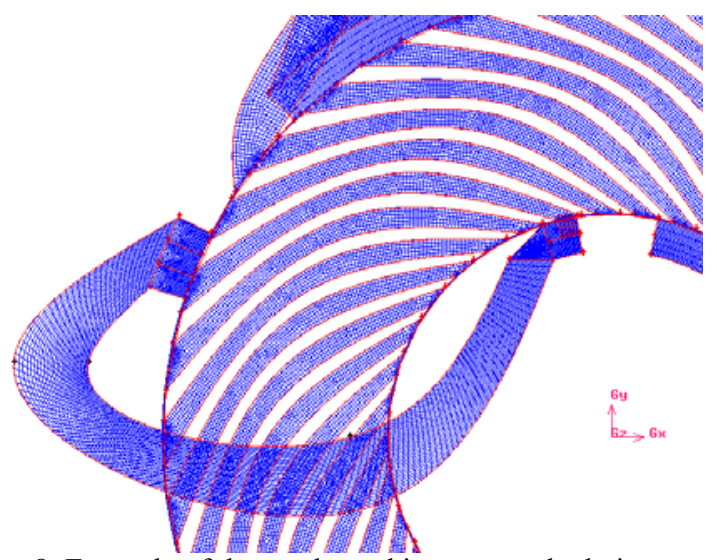

Fig. 8. Example of the mesh used in recent calculations.

The mesh used in the Fluent code can be logically separated even in the case of the geometrical overlapping flow-field zones as shown in Fig. 8.

The main advantage of the 2-D technique used was possibility to simulate many physical phenomena that could not be simulated by 1-D model. The most important is the flexibility in use of complicated cell shapes. In recent calculation, very different cell and port geometries were used. Most important is the possibility of direct simulation of the centrifugal and Corriolis forces. In addition the simulation of two-dimensional effects accompanying the cell-ports transient opening process gives the opportunity for calculation of non-predictable unsteady forces acting on the cell sidewalls. The 2-D calculations presented realize more realistic flow conditions in the case of complicated and time-variable flow-field geometries.

Fig. 8 shows how the passage connecting high-pressure hot gases port with compressed-air port through the combustion chamber zone is located over rotor zone. The proposed solution opens a possibility for using 2-D calculations of typically 3-D geometrical problems, which reduce the calculation time significantly. The paper (Piechna 2006b) showed some results of 3-D simulations. However, the time necessary for geometry and mesh preparation as well very long time required for the simulation showed that 3-D calculations were not an effective way for wave rotor investigation.

In the 2-D wave engine model considered the combustion chamber operation was simulated by the use of a model of a local heat exchanger. The heat exchanger model applied in recent simulation was a model of constant temperature heat source. Heat exchanger model is based on the following relation

$$
\begin{gathered}
H_{Q}=Q D\left(T_{\text {heater }}-T_{\text {fluid }}\right) \\
\text { where } H_{Q} \text {-heat transferred to fluid } \\
Q \quad \text {-mass flow rate of fluid } \\
D \quad \text {-heat transfer rate } \\
T_{\text {heater }} \text { - heater temperature } \\
T_{\text {fluid }} \text { - fluid temperature }
\end{gathered}
$$

Heater parameters were chosen to realize the variable heat stream - constant temperature model. The heat transfer is dependent on the actual flow rate and difference between the air flow and heat source temperatures. It is one of possible models of the combustion chamber control techniques.

In all considered cases the highest tangential rotor velocity was equal $120 \mathrm{~m} / \mathrm{s}$. Typical analysed rotor diameter was $0.12 \mathrm{~m}$ and rotational speed $2000 \mathrm{rad} / \mathrm{s}$.

\section{SINGLE STEP COMPRESSION GEOMETRY}

The simplest model of the single step compression geometry is shown in Fig. 8. Fresh air is delivered to cells on inner side of rotor while the exhaust gases are removed on the outer rotor side. Hot, high-pressure gases from the combustion chamber are delivered from the inner side and compressed air is taken away on the outer side of the rotor. The general direction of the flow is from the inner to outer rotor side. Such a flow pattern is called as through-flow configuration. Curved compression and decompression disk channels have been used to reduce the rotational velocity of the rotor.

Due to the unsteady and periodic operation of each cell the most important was the identification of phases of the torque generation. Features representing compression and decompression capabilities are similar with previous investigations in Piechna ( 2006 a, b). 

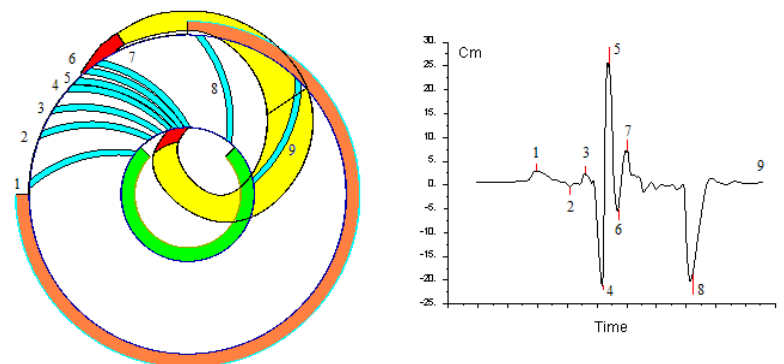

Fig. 9 Key curved compression-decompression channels positions corresponding to the characteristic points of the torque variation in wave engine in through flow configuration.

Fig. 9 shows a curve representing the temporary values of torque generated by the unsteady flow in single cell during main phases of single cycle of operation.

In the geometry considered negative torque values means that the device generates torque. The first three characteristic small peaks (1,2 and 3) in torque variation are results of the stopping the outflow of the venting air and reflection of the compression wave generated in that process. The first great peak of the torque generated (position 4) is generated by the reaction of hot gas delivered almost tangentially through the high-pressure hot-gas port. The process is unsteady, and after reflection of the primary compression wave from the closed outer cell end, the sign of torque is changed (position 5). The rotor is decelerated. Small peaks of generated torque arrive when the compressed air port opens, realizing outflow of compressed air from the cell (position 6). The torque changes sign when the inner cell end starts to be closed (position 7). The next peak of generated torque can be seen when the gas in cell expands to the atmosphere (position 8). After the gas expansion, torque changes sign and stay positive during the scavenging process (position 9). In the recent paper detailed analysis of a torque history generated in single channel was extracted and presented to explain the mechanism of the torque generation in the case of unsteady flow. Of course the total torque is the sum of torques generated by each channel. Due to the phase shift between channels the total torque variation is different and masks the way of torque generation.

Generally, an oscillatory way of torque variation can be noticed. Regarding the sign, torque is mainly generated during the opening and closing phases of the unsteady processes. Unfortunately the mean torque value in full cycle is positive, which means that device is absorbing power.

In Fig. 10 temporary pressure and temperature distribution data are presented. Presented pressure is a gauge pressure and operating pressure is $100000 \mathrm{~Pa}$. General flow direction inside the rotor is from the inner side to the outer side.

Another configuration, called reverse flow configuration, was also analyzed. As previously, the fresh air is delivered on the inner rotor side and exhaust gases removed on the outer side, but the port delivering hot, high pressure exhaust gases from the combustion chamber was located on the outer and compressed air port at inner side of the disk.
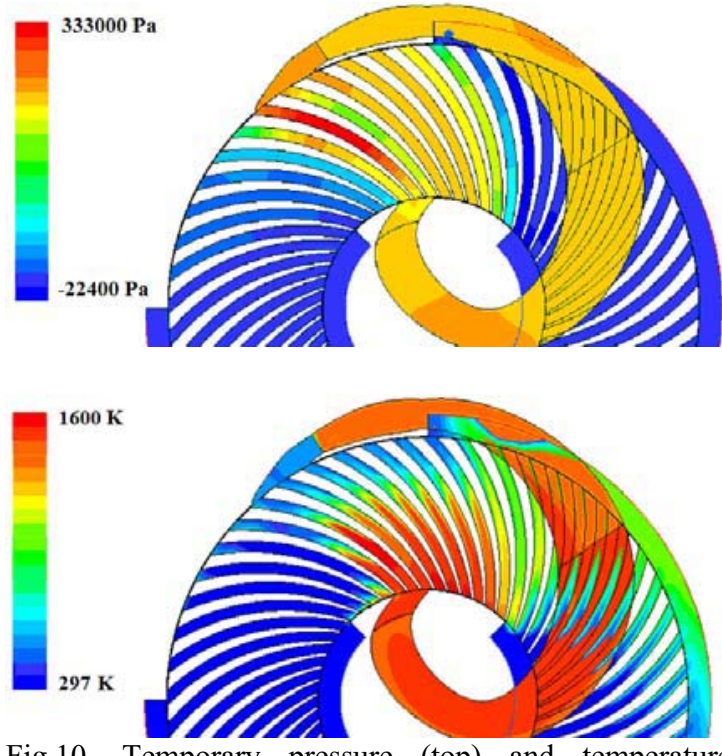

Fig.10. Temporary pressure (top) and temperature (bottom) distribution in simple through flow wave engine configuration.
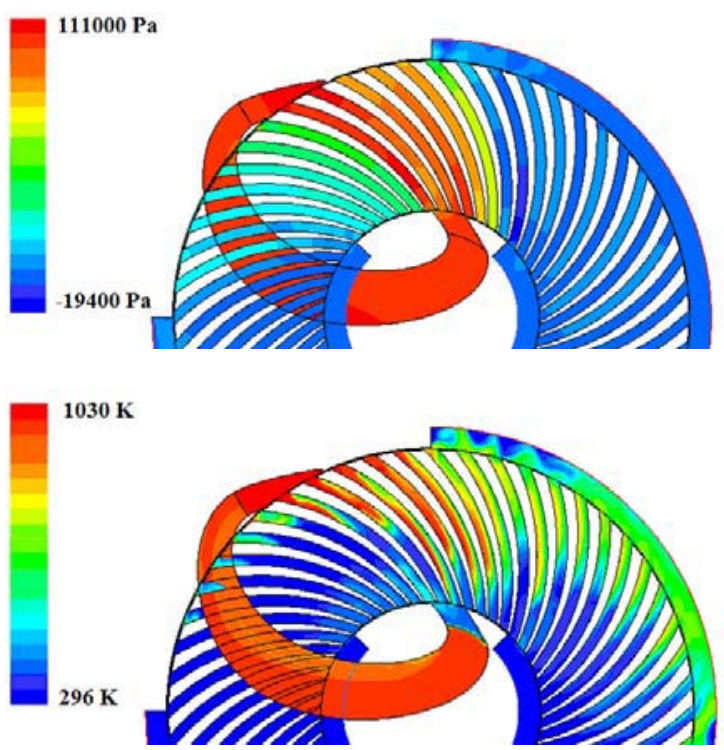

Fig. 11. Temporary pressure (top) and temperature (bottom) distribution in simple reverse flow wave engine configuration.

Fig. 11 presents the temporary pressure and temperature distribution. It shows that it is possible to compress in unsteady way the air by the hot exhausted gases even against the action of centrifugal forces. Due to relatively short compression-decompression channels the time of unsteady processes is very short, and unsteady expansion process is very rapid.

Few different forms of shapes of disk channels and ports have been investigated. Results of calculation of pressure and temperature for disk channel geometry with straight, oblique and variable widths in reverse and through-flow configuration are shown in Figs. 12 and 13. The most promising results have been obtained with the shape of disk channels and port geometry shown in Fig. 14. 


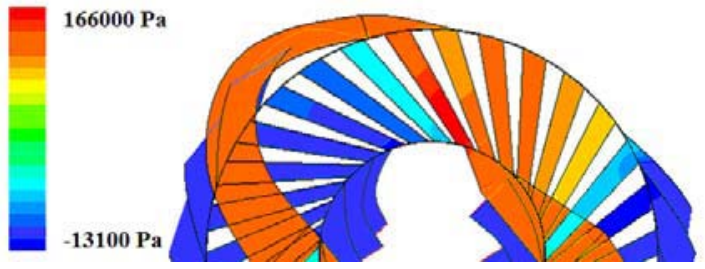

$1520 \mathrm{~K}$

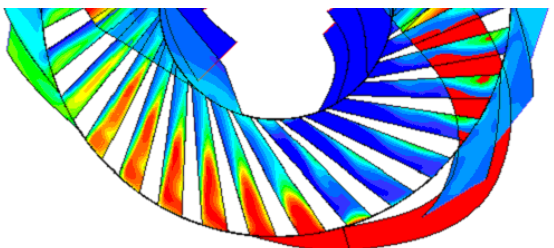

Fig. 12 Pressure (top) and temperature (bottom) distribution in reverse flow configuration (straight, oblique channels with variable cross section)
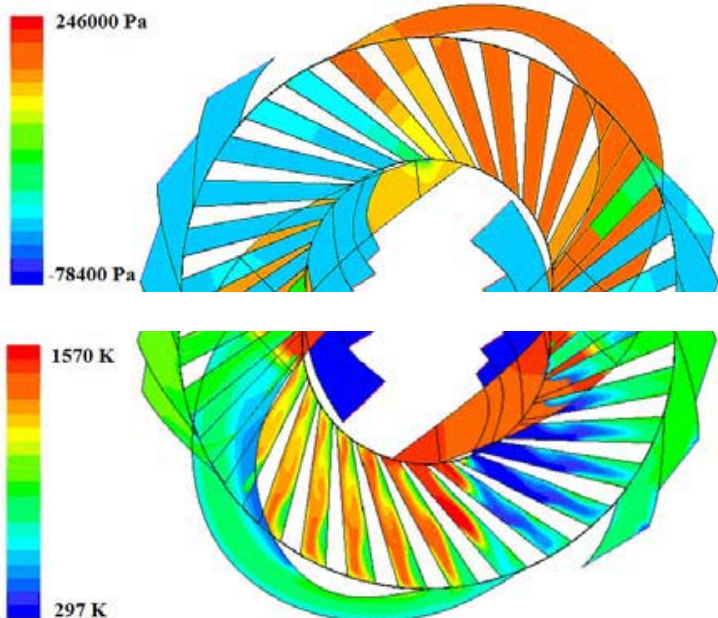

Fig. 13 Pressure (top) and temperature (bottom) distribution in through flow configuration (straight, oblique channels with variable cross section)
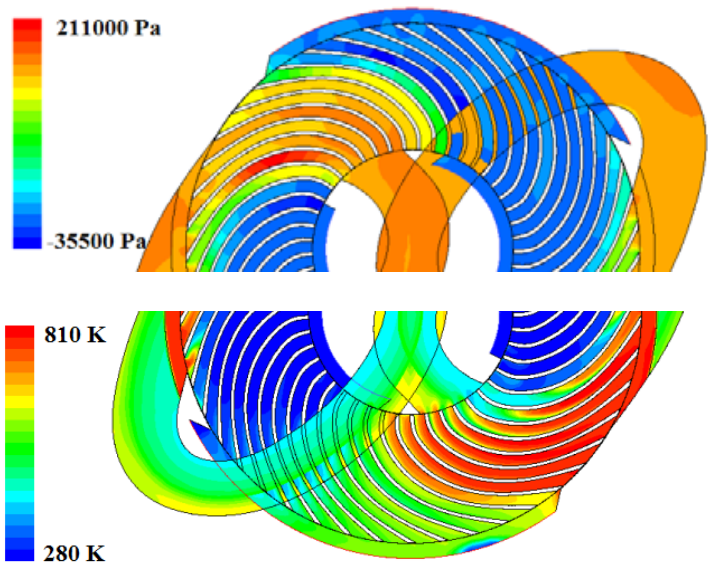

Fig. 14. Temporary pressure (top) and temperature (bottom) distribution inside wave engine with double set of ports

Figure 14 shows rotor and port geometry with a double set of ports. The wave engine with this geometry worked in a stable manner and generated torque. However, the thermodynamic efficiency was on the level of $2 \%$ only.

\section{RECIRCULATION BYPASS FOR TORQUE GENERATION}

As mentioned above, while the unsteady process is useful for the compression, the steady state is more efficient for decompression. Accordingly an attempt was made to use a steady type of decompression. Modifications of the port geometry were made to add the new channel for a realization of the two-step decompression and to use the same disk for torque generation.
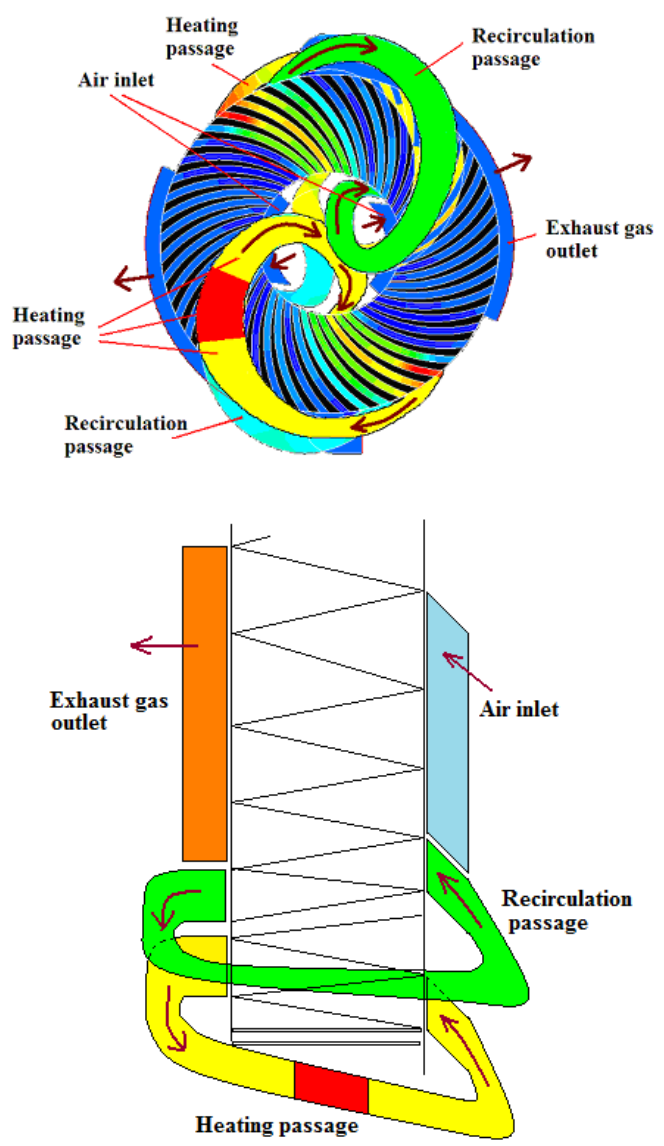

Fig. 15. Wave engine configuration with additional recirculation passage for torque generation.

A schematic representation of the flow is shows in Fig. 15. After delivering compressed air to the combustion chamber (heating passage), air in disk channel is under relatively high pressure to pass it through the recirculation passage to the inner side of the disk, realize the momentum change entering the disk channel and push the exhaust gases to the outlet. In that way air is passing the disk channels twice.

The pressure and temperature distribution for such a configuration is presented in Fig. 16. Unfortunately, records of the torque values did not confirm expectations. The mean value of the torque showed that the power was delivered to the rotor instead of being generated. It is worth be noticing that the main flow direction in the geometry considered was from the inside to the outside of the rotor, which is opposite to the typical steady-state machines intended to generate torque.

A similar idea was realized in a slightly different geometry. Fig. 17 shows a pressure and temperature distribution for the coupled set of ports. Existing in 
original geometry low frequency oscillations have been reduced in this coupled version.
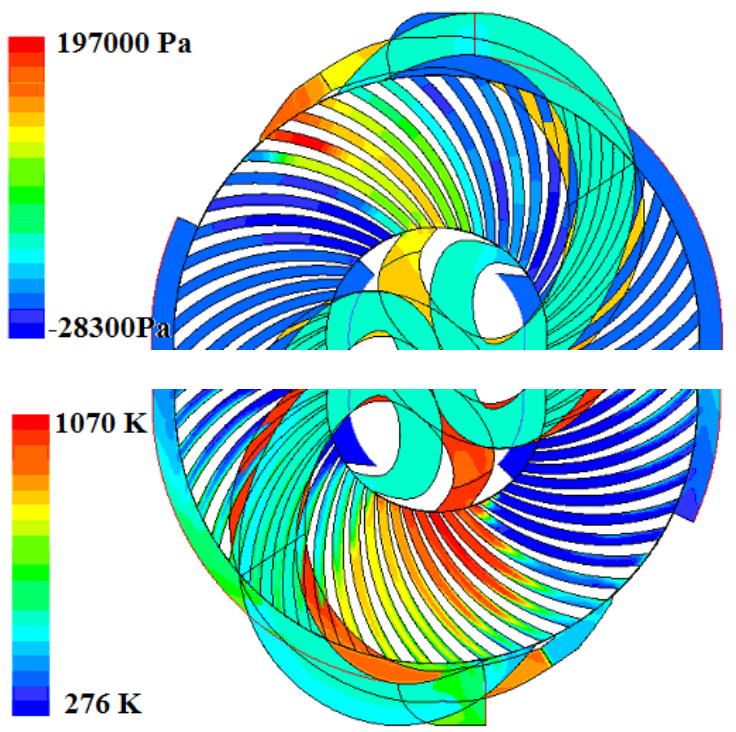

Fig. 16. Pressure (top) and temperature (bottom) distribution in wave engine configuration with additional recirculation passage for torque generation and scavenging improvement.

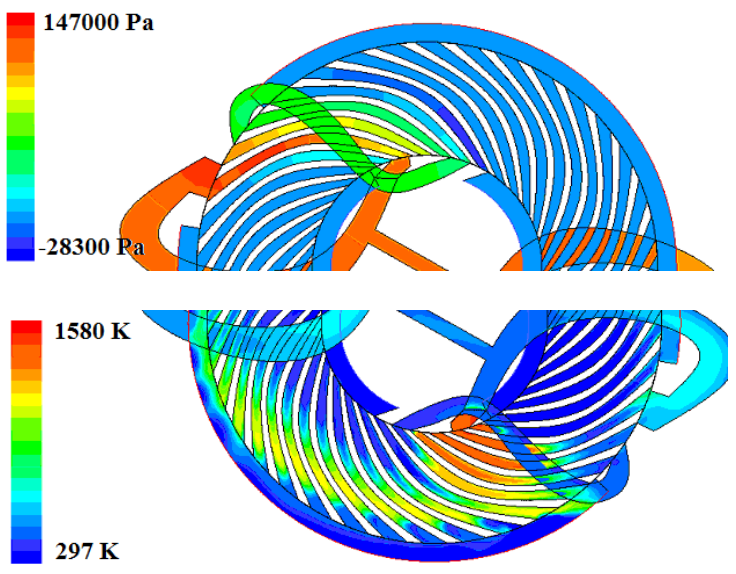

Fig. 17. Additional passage realizing the two-step decompression and improving the scavenging.

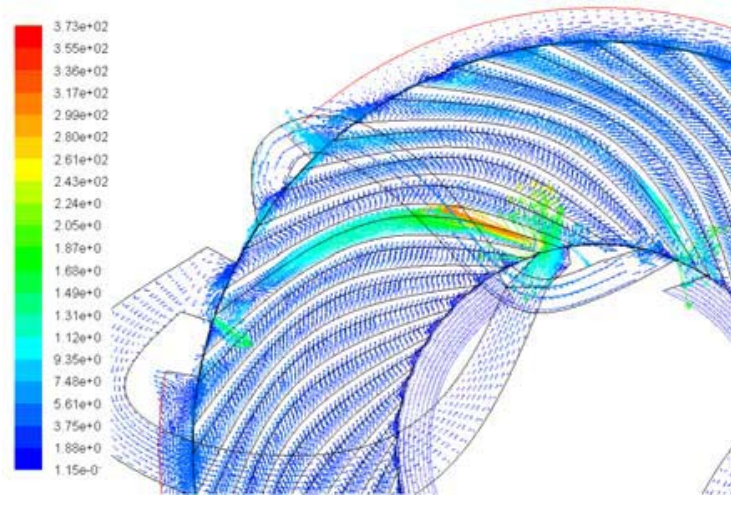

Fig. 18. Particulars of the velocity distribution in vicinity of ports.

Particulars of the velocity field near the ports are visualized in Fig. 18. Hence the changes of the velocity directions in inlets to the compression-decompression disk channels are clearly visible confirming the momentum change and torque generation. Torque generation is a highly unsteady process where phases of torque generation are followed by phases of disk deceleration.

\section{TWO-STEP COMPRESSION GEOMETRY}

Two-step compression wave engine configuration was considered. From the previous 1-D calculations (Piechna 2006) it was known that wave engine operation in such conditions is rather unstable. The main problem in 2-D simulations is very long time of calculation. Any modifications of geometry or flow parameters require a very long time of computer work to yield the results.

In Fig. 19 scheme of flow in considered geometry is presented. The main idea is the following: Fresh air entering the wave engine is precompressed (first step) by the medium pressure gases from the decompressionprecompression passage. The main compression (second step) is realized by the high-pressure hot gases from the heating passage (containing combustion chamber). Air after two steps of compression is delivered to the combustion chamber and heated. High-pressure hot gases after compressing second time the air are decompressed partially to the decompression-precompression passage. Later are removed to the atmosphere in the final decompression process.

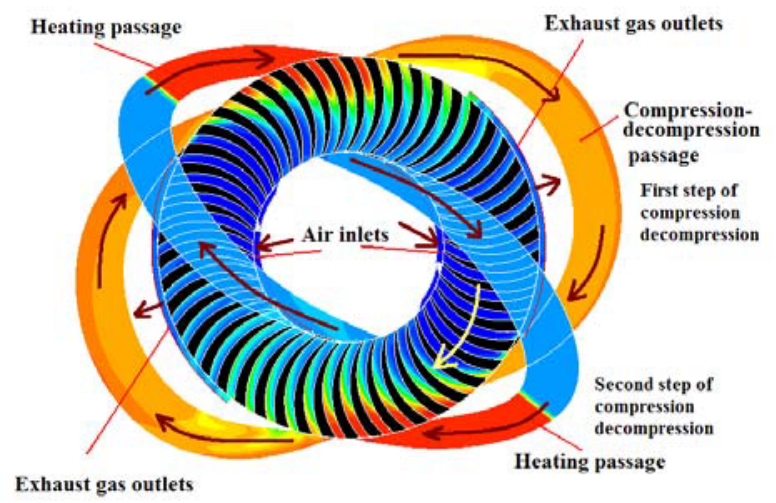

Fig. 19. Scheme of the passage configuration for the twostep compression-decompression wave engine geometry

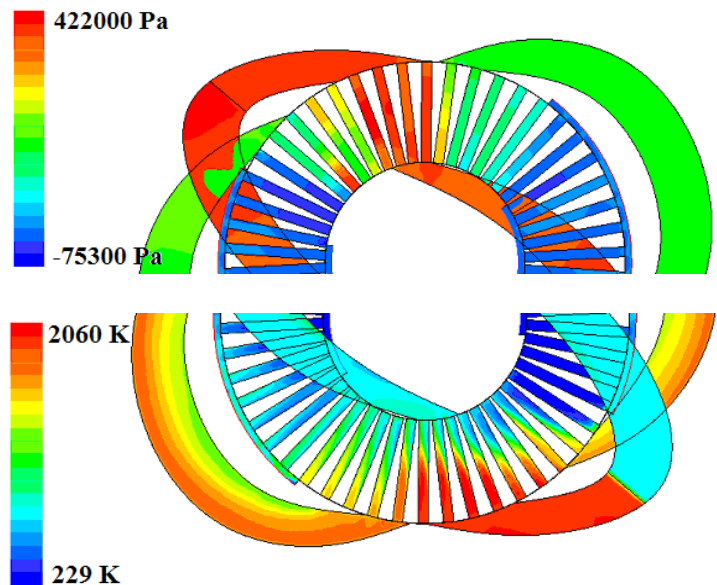

Fig. 20. Pressure (top) and temperature (bottom) distribution inside the wave engine with two-step compression- decompression geometry. 
Pressure distribution presented in Fig. 20 shows the total compression ratio of $5: 1$ (pressure in combustion chamber to air inlet pressure). In Fig. 20 (bottom) the corresponding temperature distribution is depicted.

Details of the velocity field are shown in Fig. 21 clarifying the two-step compression idea.

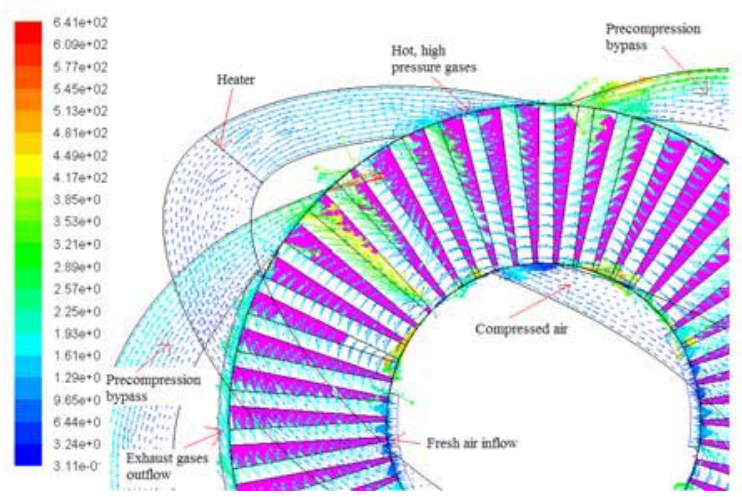

Fig. 21. Details of the velocity field inside the wave engine with two-step compression- decompression.

\section{CONCLUSIONS}

A wide range of different geometrical disk wave engine configurations were analyzed. Due to purpose of further miniaturization of device, the wave disk geometry (radial displacement of the disk compression-decompression channels) was considered as a main wave engine configuration. In radial scheme, the disk requires a wider diameter, but it can be flat and thin. The wave engine design investigation presented in this paper was concentrated on two main problems: realization of gas compression and simultaneously a torque generation. Only pure unsteady way of operation was considered. From the point of view of efficiency of unsteady processes the length of the compression-decompression channels should be ten times greater than the channel width. Such a proportion is necessary to reduce the influence of the finite time of the channel opening process.

For the torque generation, a high ratio of channel length to width is not very useful, particularly for constant width channels. Curved channels have been used to increase their real length and reduce the rotor rotational speed required for synchronization. The rotational speed of a disk is three times lower than for comparable steady-state machines. Also top linear speed of rotating elements is three times lower than steady-state machines. Compression in such conditions is more effective in unsteady way but decompression is not. Locally, the gas speed reached $300 \mathrm{~m} / \mathrm{s}$, but generally was much lower. Due to the unsteady flow conditions the torque strongly varies in time.

In conditions where the unsteady processes are mixed with known from steady state machines actions of centrifugal and Corriolis forces, predictions of final results are rather difficult. Unsteady process of gradual channel ends opening, yields in 2-D model, information about the additional unsteady forces existing due to the non-uniform pressure distribution acting on the disk. Unfortunately, experience collected in design of steadystate machines cannot be very useful in case considered. The main problem recognized in wave engine operation is still related to bad scavenging possibilities. Due to the relatively low rotational speeds the centrifugal forces are not very strong but in many analyzed cases effectively realized the scavenging process. The scavenging process in such conditions occupies the great part of the disk space. Therefore, the energy density related to the disk volume is relatively low.

A wave disk can be an effective compression device and in conjunction with the combustion chamber can form an efficient gas generator but a wave engine design on the basis of only unsteady processes cannot be an efficient device. In specific wave-disk geometry, torque generation is possible, but it is not as effective as in the case of steady-state machines.

\section{REFERENCES}

Akbari, P., "Performance Prediction and Preliminary Design of Wave Rotors Enhancing Gas Turbine Cycles," Ph.D. dissertation, Michigan State University, East Lansing, MI 2004

Epstein A.H., "Millimeter-scale, MEMS gas turbine engines," GT-2003-38866,Proc. of ASME Turbo Expo 2003, June 16-19, 2003, Atlanta, GA, 2003

Frechette L.G., "Development of a Microfabricated Silicon Motor-Driven Compression System," Ph.D. dissertation at MIT, Cambridge, MA, 2000

Hörler H.U., „Abschätzung der Verluste in InstationärGasdynamischen Kanal-Trommel-Drucktauschern." Ph.D. thesis No 4402, ETH Zurich, Switzerland, 1969

Iancu F., Piechna J., Dempsey E., Müller N., "Ultra-Micro Wave Rotor Investigations," Technical Digest PowerMEMS 2005, The Fifth Int. Workshop on Micro and Nanotechnology for Power Gen. and Energy Conv. Appl., 2005 Tokyo, pp. 93-96

Pearson R. D., „A Gas Wave-Turbine Engine Which Developed 35 HP and Performed Over a 6:1 Speed Range, " Proc. ONR/NAVAIR Wave Rotor Research and Technology Workshop, Report NPS-67-85-008, pp. 40-49, Naval Postgraduate School, Monterey, CA, 1985

Piechna J., Akbari P., Iancu F., and Müller N., "Radial-flow wave rotor concepts, unconventional designs and applications," IMECE2004-59022, 2004

Piechna J., "Wave Machines, Models and Numerical Simulation”, Oficyna Wydawnicza Politechniki Warszawskiej, Warszawa, 2005

Piechna J., "Feasibility Study of the Wave Disk MicroEngine Operation," Technical Digest PowerMEMS 2005, The Fifth Int. Workshop on Micro and Nanotechnology for Power Gen. and Energy Conv. Appl., 2005, Tokyo, pp. 69-72.

Piechna J., "The micro jet wave engine idea," The $2^{\text {nd }}$ International Symposium on Innovative Aerial/Space Flyer Systems (Dec. 2-3, 2005 The University of Tokyo) PL-12, pp. 71-78

Piechna J., "Numerical Study of the Wave Disc MicroEngine Operation", The Archive of Mechanical Engineering, vol. XLV, Nr. 4, pp. 305-323, 2006

Piechna J., 2006, "Feasibility study of the wave disk microengine operation", Journal of Micromechanics and Microengineering, 16 (2006), pp. 270-281.

Ribaud Y., Dessornes O., Guidez J., Courvoisier T., Dumand C., Kozanecki Z., Helin P., Le Moal P., Minotti P., "The experience gained on the ultra microturbine: from energetics to component brick studies," Technical Digest PowerMEMS 2005, The Fifth Int. Workshop on Micro and Nanotechn. for Power Gen. and Energy Conv. Appl., 2005, Tokyo, pp. 21-24

Schneider B., Bruderer M., Dyntar D., Zwyssig C., Diener M., Boulouchos K., Abhari R.S., Guzzella L., Kolar J.W., „Ultra-High-Energy-Density Converter for Portable Power," Technical Digest PowerMEMS 2005, The Fifth Int. Workshop on Micro and Nanotechn. for Power Gen. and Energy Conv. Appl., 2005, Tokyo, pp. 81-84.

Weber H. E., 1995, „Shock Wave Engine Design“, New York: John Wiley \& Sons. 